$\mathbb{T}$ periodica polytechnica

Mechanical Engineering

$54 / 1(2010) 41,48$

doi: 10.3311/pp.me.2010-1.07

web: http://www.pp.bme.hu/me

(c) Periodica Polytechnica 2010

RESEARCH ARTICLE

\section{Fuzzy evaluation of statistically based generalized mechanical functions}

\author{
Attila Piros / Tibor Bercsey
}

Received 2011-01-18

\begin{abstract}
In mechanical design engineers many times have to find the kind of parameters which are not known but must be realistic. To make this case even harder sometimes ambiguous parameters have to be evaluated. This paper introduces two mathematical methods to find the optimal parameters of the mechanical functions. Fuzzy method helps the evaluation of the mechanical functions and their combinations. The statistically based generalized function models provide realistic input for this fuzzy evaluation. Both methods are easy to convert into algorithm and they are tested in an up to date design task.
\end{abstract}

\section{Keywords}

Fuzzy evaluation $\cdot$ generalized mechanical functions $\cdot$ data interpolation

\section{Attila Piros}

C3D Engineering Consultant Ltd., Isaszegi ut 76. Pecel, 2119, Hungary e-mail: piros@c3d.hu

Tibor Bercsey

Department of Machine and Industrial Product Design, BME, H-1111 Budapest, Múegyetem rkp 1., Hungary

e-mail: bercsey.tibor@gt3.bme.hu

\section{Introduction}

Nowadays engineers have to work with high complexity mechanical models. It is a difficult task to find the good parameters of a non-existing construction or to select the proper combinations of the existing components. Due to the strict deadlines and the high variety of parameters and components, advanced mathematical methods need to be introduced to help the engineers in the design procedure. This paper describes a fuzzy evaluation method which, if applied on generalized mechanical models, helps the designers to find the proper values of the required design variables. The generalized mechanical functions are based on statistically preprocessed data of realized mechanical components.

These functions in the design model can simulate the main components of the construction. These simulated components produce different mechanical properties on these outputs. These properties are further processed with fuzzy evaluating and compatibility check. The compatibility functions define taboo zones to radically decrease the calculating time. Fuzzy evaluation helps to find the best combinations also in ambiguous cases.

In a design process engineers usually try to find the optimal solution of a specific problem. In the case described in this paper the optimal solution means the best combination of the components. The quality of a specific combination depends on the parameters of the selected components. If the selection is only based on existing and well known components probably some good combination would be missed. The statistically based generalized mechanical functions eliminate this problem because these mathematical models use interpolated data therefore they cover the whole solution space.

The mechanical properties calculated from the generalized mechanical functions are tested with compatibility functions to filter the incompatible combinations. After this initial check the acceptable combinations are further processed and evaluated. With traditional evaluation specific components may be excluded because of these properties, e.g. sometimes a value of a property is out of the traditional accepted range but this component can be a member of the optimal combination. Fuzzy evaluation helps to avoid ignoring this kind of component. 
Fuzzy method enables handling cases when a parameter is not obviously in a good range. Special functions (fuzzy membership functions) can be defined to mathematically handle these cases.

\section{Fuzzy evaluation in mechanical conceptual design}

Fuzzy logic handles the ambiguous cases of parameter evaluation. This way is close to human thinking because by answering some simple questions the concerning fuzzy membership functions can be set up. A typical fuzzy membership function results on its output a number between 0 and 1 . Zero means that the studied value is totally outside the set or range. 1 marks that the value is totally inside the range. Any other value between 0 and 1 shows the ratio of the membership.

There is a servo motor with a given speed-torque characteristic. There is a required torque from the load side of the construction. The evaluation of this case is not as simple as it seems at first sight. The motor can produce more torque than its maximum torque in overloaded case (see Fig. 1).

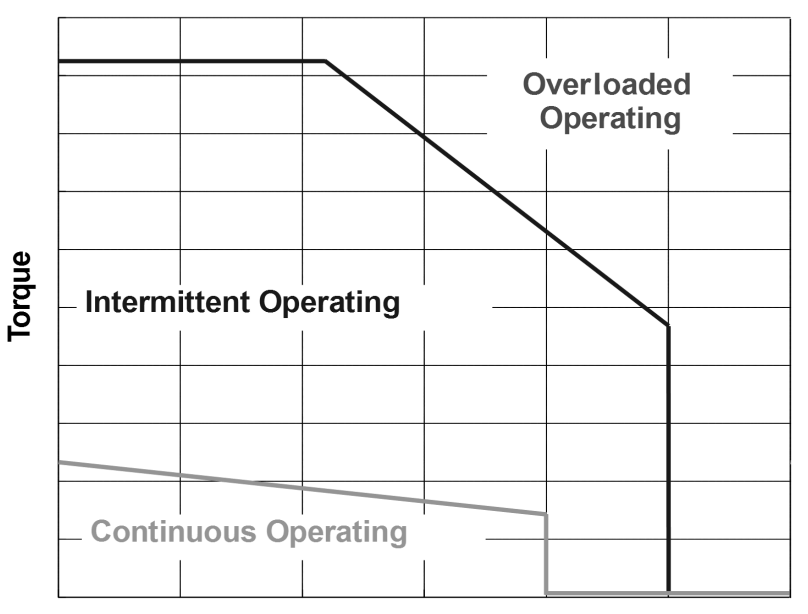

Speed

Fig. 1. Speed-torque characteristic of a specific servo motor

Fuzzy membership functions can handle this special case of overload very well (see Fig. 2). The decreasing fuzzy value after the maximum torque simulates the case of overload well.

The evaluation needs special preparation to have proper results at the end of the procedure. Besides establishing the fuzzy membership functions, the design properties must also be classified. There are two kinds of design properties. One of them depends on one or more design variables. The utilization factor of the motor can be a good example for a typical variable property. Some other properties are independent of any design variables. The size and the price of the components are typical examples for the invariant design property.

The two kinds of properties require different handling during the evaluating process. Both need to be weighted and normalized because of the fuzzy membership functions. These functions get results between zero and one, therefore the property

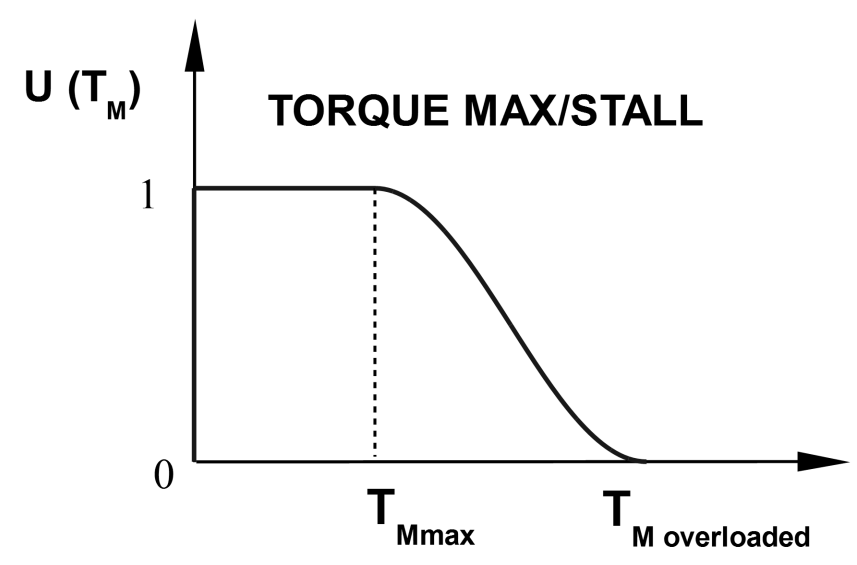

Fig. 2. Fuzzy membership function of a specific servo motor

values must also fit into this scale.

At the first step the properties are weighted on a range from one to ten. Then in case of the parameter variant properties the weights are transformed to an $\left(R W_{\min }-R W_{\max }\right)$ range. The (0.5-1) range provides the best data distribution based on the competed studies. A specific $W_{V A R_{i}}-W^{\prime} i_{V A R_{i}}$ weight transformation is calculated with the following formula, where the $W_{V A R_{i}}$ weights are categorized in a (0-10) scale.

$$
\begin{gathered}
W_{V A R}^{\prime} i=R W_{\max }-\frac{R W_{\max }-R W_{\min }}{10} \cdot W_{V A R} i \\
R W_{\max }=1 R W_{\min }=0.5 \\
1 \leqslant W_{V A R} i \leqslant 100<W_{V A R}^{\prime} i \leqslant 1
\end{gathered}
$$

Before weighting the invariant properties the range of a specific property must be established. The invariant weights are normalized and distributed on this $P_{I V \text { min }}-P_{I V \text { max }}$ range. Weighting and normalizing the parameter invariant properties are based on the following formula:

$$
\begin{aligned}
& W_{I V j}=\frac{\frac{P_{I V \max }-P_{I V j+1}}{P_{I V \max }-P_{I V \min }} \cdot 9}{10} \\
& 0.1 \leqslant W_{I V j} \leqslant 1 \quad P_{I V \max }, P_{I V \min }, P_{I V j} \in \mathbb{Z} \\
& (\mathbb{Z}: \text { set of integers) }
\end{aligned}
$$

In this stage all possible configurations are automatically generated to cover the whole design space. These configurations are based on the later described generalized mathematical models. The different functions or virtual components are created by varying the parameters of the generalized models. The number of the combinations depends on the density of the parameters. The smaller steps between two neighbouring values result higher density. The maximum of the achieved density is limited by the available computing capacity.

Generating means that the components are simply paired without any tests. Although this generation procedure is quite fast, further evaluation requires many more related calculations. 


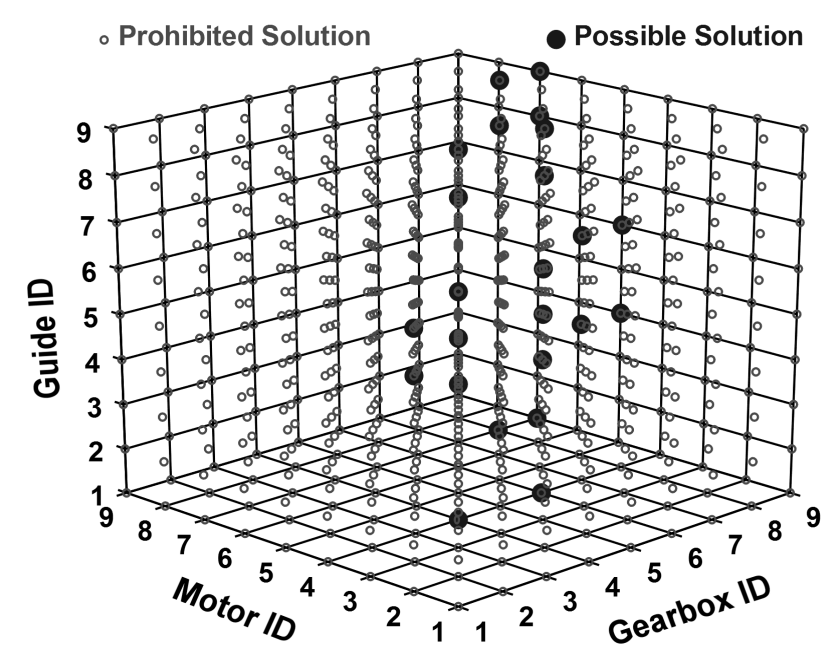

Fig. 3. Taboo zones in the solution space

By using special prohibited areas (so called taboo zones) the number of possible valid sets are significantly decreased. Fig. 3 shows the taboo zones in a selected subset.

In case of connecting components the taboo zones are defined by compatibility functions. A typical compatibility function compares the concerning parameters at the two connecting components. A $C P_{i}$ compatibility function is a logical function with the required parameters $\left(a_{i} b_{i} \ldots x_{i}\right)$ on its input. The function itself uses simple relations to decide on the compatibility of the given inputs (e.g. do the shafts' dimensions match or not).

$$
C P_{i}\left(a_{i} b_{i} . . x_{i}\right)=f\left(a_{i} b_{i} . . x_{i}\right) \quad C P_{i} \in[01]
$$

After filtering the solution space with taboo zones many other $P_{V A R n}$ variant parameters of the created configurations must be calculated. The $u_{V A R i}$ fuzzy values are defined with the concerning $U_{i}$ fuzzy membership functions:

$$
u_{V A R i}=U_{i}\left(P_{V A R} i\right)
$$

There are many different functions that must be evaluated in the solution space. Generally the number of the handled fuzzy membership functions are too big to unify them. Literature has examples of unification of only two or three functions. Usually in practice there are five or more membership functions in a simplified case. The different design variations need to have a single, quality related value for comparison.

Introducing the corrected fuzzy mean $\left(R_{F Z}\right)$ there is a good opportunity to summarize as many results of the fuzzy membership function as it is required to evaluate the specific design. This value is much simpler to calculate than the traditional results of the fuzzy inference systems.

$$
R_{F Z}=\frac{u_{V A R} 1^{W_{V A R}^{\prime} 1} \cdot u_{V A R} 2^{W_{V A R}^{\prime} 2} \cdot \ldots \cdot u_{V A R} n^{W_{V A R}^{\prime} n}}{\frac{\sum_{i=1}^{n} u_{V A R} i}{n}}
$$

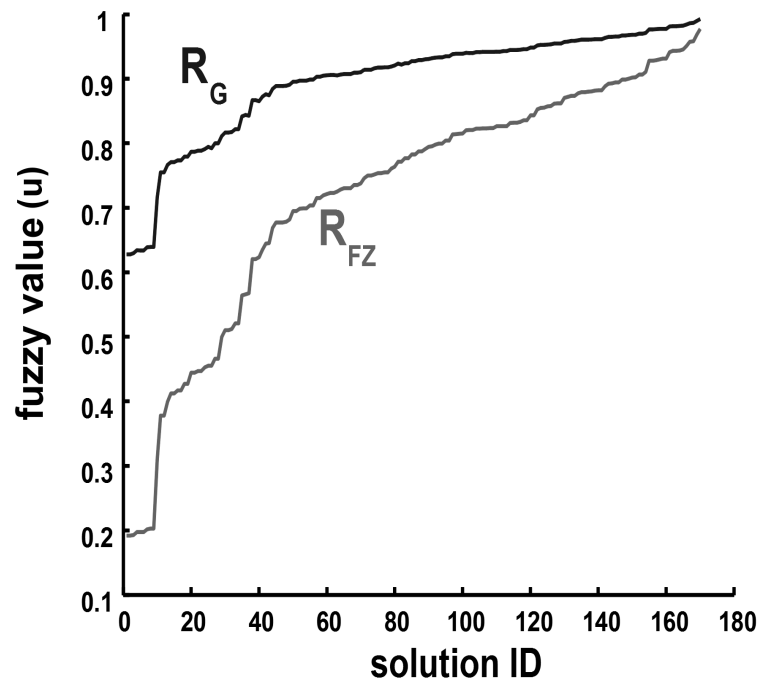

Fig. 4. Distribution of mean values

The corrected fuzzy mean is similar to the geometric mean $\left(R_{G}\right)$.

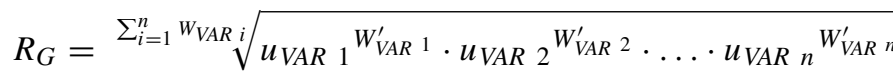

Both means meet the two fundamental criteria $(C 1 C 2)$. The first criteria says that if any fuzzy value is equal to zero the mean value must be zero. In this case this variation is out of the range because of the failure of one of its parameters.

$$
C 1: 0 \in u_{V A R} i \rightarrow R_{i}=0
$$

The second criteria concerns the ideal case. If all of the parameter values are equal to one the calculated mean must also be one.

$$
C 2: \frac{\sum_{i=1}^{n} u_{V A R} i}{n}=1 \rightarrow R_{i}=1
$$

The main difference between these means is the distribution of the mean values. The values of the geometric mean are distributed on a narrower range than the values of the corrected fuzzy mean. Fig. 4 shows that the corrected fuzzy mean separates the design variations better than the geometric mean.

The evaluated design variation is unequivocally characterized by using the corrected fuzzy mean. The $Q_{V A R}$ variant quality value is calculated from the related fuzzy values in each design variation.

$$
Q_{V A R i}=R_{F Z}\left(u_{V A R} i\right)
$$

As the final step the invariant properties must be considered by the calculated invariant weights. The variant quality weighted with the $W_{I V}$ invariant weights results the $Q_{U T}$ united quality value.

$$
Q_{U T i}=W_{I V i} \cdot Q_{V A R} i
$$


The highest $Q_{U T}$ value marks the best design among the different variations.

\section{Generalized mechanical functions}

During the conceptual design many different physical principles and mechanical functions are handled. The following section introduces a statistically based method to create mathematical models for these functions.

The statistically based model is established on analyses of the existing solutions. The aim of this method is to create a usable model without knowing the exact principles and the inside relations of the function. The method statistically analyses many instances and fits generalized functions to the results. The mathematical model is based on these functions. The different variations of a function are resulted by modifying this model.

In case if there is only one dimensional parameter that describes a property the creation of the generalized function is quite obvious.

In the following example the objective is to create the generalized function of friction torque $\left(T_{F R}\right)$ for servo motor. In this simplified case there are three different values of the friction torque for three different servo motors characterized by different inertia values. Fig. 5 shows the result of fit.

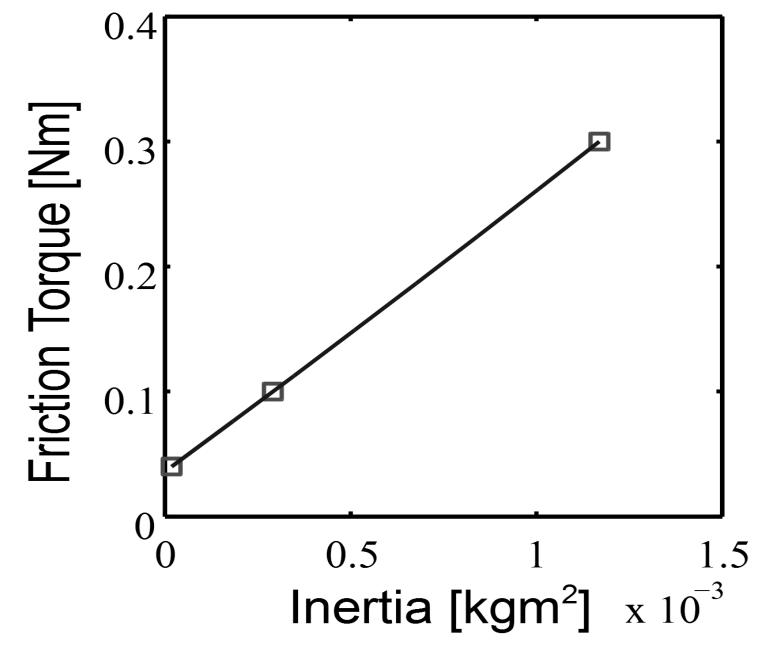

Fig. 5. Generalized inertia - friction torque function

Some of the parameters of the existing components are not one dimensional. The typical two dimensional properties are displayed in characteristic diagrams. The three dimensional extension of these diagrams results the generalized function for this case. The previous servo motors have several speed-torque diagrams (see Fig. 6). The building of the generalized speedtorque function requires the following steps.

STEP 1. The diagram must be sampled for further processing (see Fig.7).

STEP 2. Other digitized diagram is placed in the three dimensional space concerning the inertias of the motors (see Fig. 8).

STEP 3. Intermediate data is generated using interpolation to estimate the non-existing values (see Fig. 9).

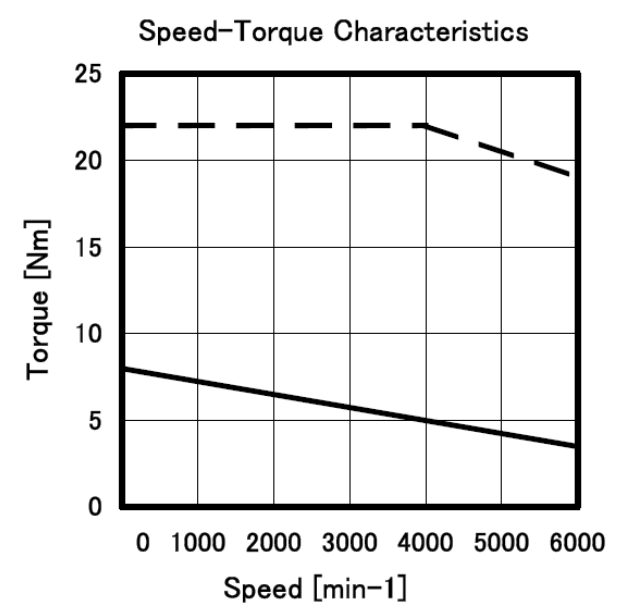

Fig. 6. Typical characteristic diagram

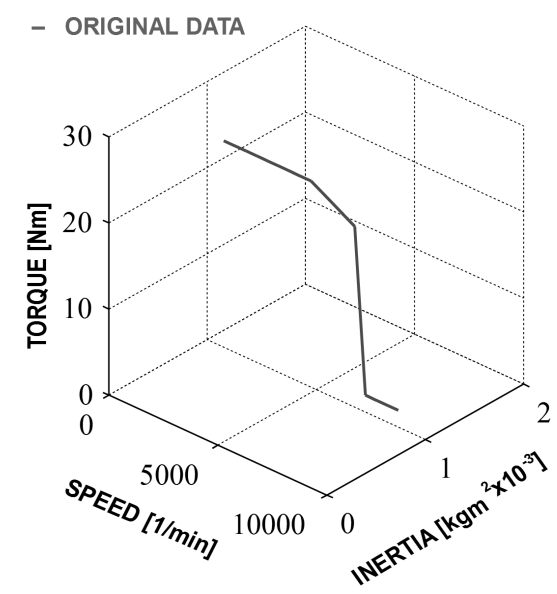

Fig. 7. Sampled speed-torque diagram

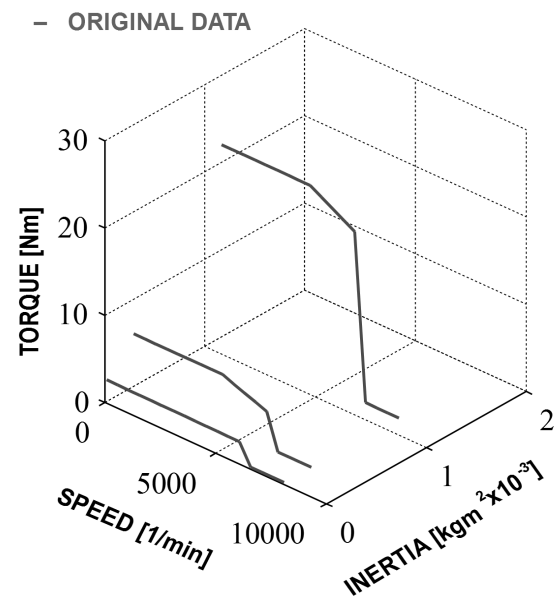

Fig. 8. The original dataset

The previously described examples use polynomial fitting to produce the generalized functions.

$$
y=p_{1} x^{n}+p_{2} x^{n-1}+\ldots+p_{n} x+p_{n+1}
$$

Although in these ultimately simplified cases the polynomial fitting gives satisfying results, in other cases different method is required. In case of higher number of initial data the spline 


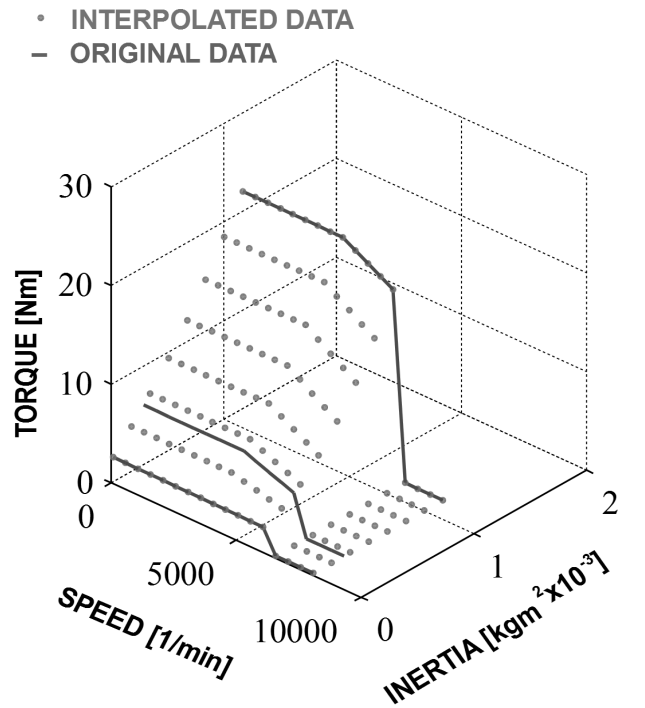

Fig. 9. The generalized speed-torque function with interpolated data

interpolation has to be considered.

Some of the parameters are totally out of the range of the existing data. A typical case of this kind of theoretic functions is when there is a direct connection between a motor and a guide instead of applying intermediate gearbox and coupling. Both the gearbox and the coupling have specific limited parameters (inertia, maximum torque, etc.). But an idealistic component usually has extreme data values (zero inertia, infinite maximum torque, etc.). These kinds of parameters require special handling. These need to be separated from the other data therefore the interpolation results in inappropriate functions.

The generating of the design parameters are quite simple using the statistically based mathematical models. All of the parameters can easily be calculated with the generalized functions. In this way the whole design space can be fully covered. For the previously described servo motor a calculated torque value is displayed on Fig. 10 .

\section{Case study: design of linear drive system}

This paper introduces a typical case study of mechanical design. In this case the task is to find the appropriate parameters for the components of a linear drive system. The arrangement of this robot motion system is shown on Fig. 11

The robot itself is integrated into a tool magazine. There are hundreds of milling tools stored in this magazine and the robot manages the tool transfer inside the magazine, see Fig. 12 .

This example is only limited to the calculation and evaluation of the variant properties. In this case these properties are listed in Table 1.

First the required torque value $\left(T_{C L}\right)$ is calculated at the load side then this value is recalculated to the servo motor side $\left(T_{C M}\right)$ with the following functions:

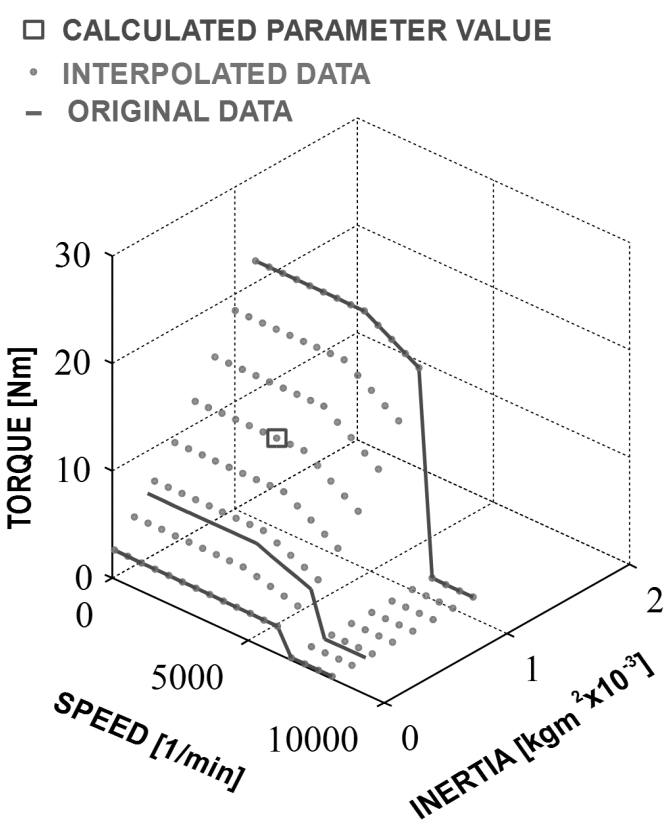

Fig. 10. A calculated torque at a specific speed-torque value

Tab. 1. The variant properties in the design process

\begin{tabular}{ll}
\hline Name & Description \\
\hline speed $\left(v_{M}\right)$ & $\begin{array}{l}\text { Speed of the moving load at the end of the } \\
\text { kinematic chain. }\end{array}$ \\
\hline inertia ratio $\left(R_{I L}\right)$ & $\begin{array}{l}\text { Ratio between the reflected load inertia and } \\
\text { the motor inertia. }\end{array}$ \\
\hline maximum torque $\left(T_{a M}\right)$ & $\begin{array}{l}\text { The required torque at the motor shaft in } \\
\text { case of acceleration. }\end{array}$ \\
\hline stall torque & The required torque at the motor shaft in \\
& case of constant velocity. \\
\hline utilization ratio & Ratio between the motor maximum torque \\
& and the required acceleration torque. \\
\hline
\end{tabular}

$$
\begin{aligned}
T_{C L}=m_{L} \cdot g & \\
T_{C M}=\frac{T_{C L}}{i_{G B} \cdot \eta}+ & T_{f r} M \\
m_{L} & : \text { mass of load } \\
g & : \text { gravity const. } \\
i_{G B} & : \text { gearboxratio } \\
\eta_{G B} & : \text { gearboxefficiency } \\
T_{f r} M & : \text { friction torque of motor }
\end{aligned}
$$

Knowing the kinematic properties the reflected load inertia $\left(I_{R L}\right)$ and the inertia ratio at the motor side $\left(R_{I L}\right)$ have to be calculated:

$$
\begin{aligned}
& I_{R L}=\left(\frac{m_{L} \cdot D P R_{G}}{2 \cdot \pi}\right)^{2}+I_{G B}+I_{C} \\
& R_{I L}=\frac{I_{R L}}{I_{M}}
\end{aligned}
$$




\section{B SLIDE + TOOL}

$2: 5$

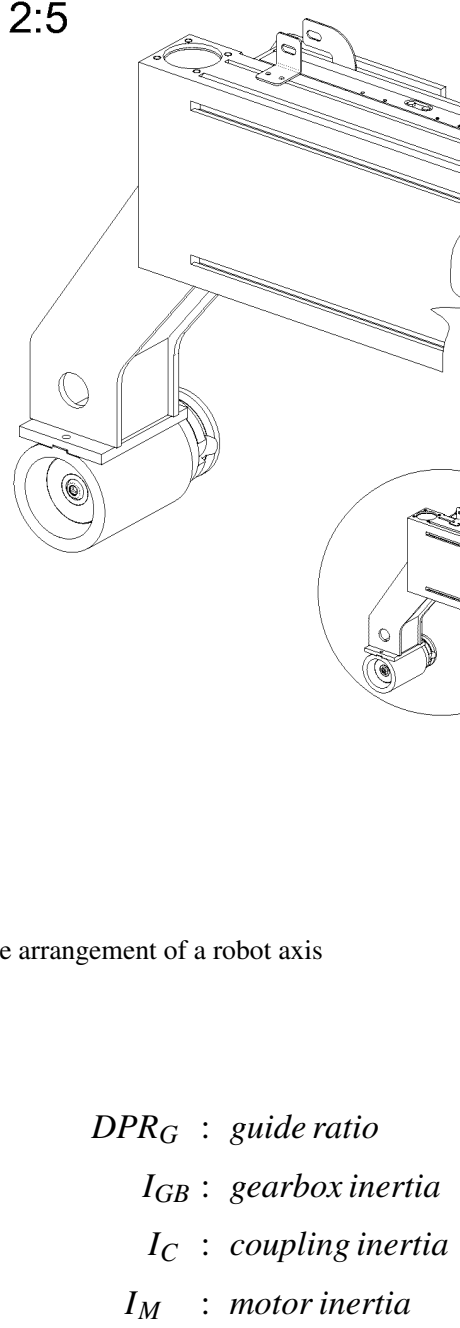

Concerning the required acceleration torque at the load side $\left(T_{a L}\right)$ and the gearbox ratio the total acceleration (maximum) torque at the motor side $\left(T_{a M}\right)$ can be calculated with these functions:

$$
\begin{array}{r}
T_{a L}=v_{M} \cdot \frac{2 \cdot \pi}{t_{a}} \cdot\left(I_{M}+\frac{I_{R L}}{\eta_{G B} \cdot \eta_{G}}\right) \\
T_{a M}=T_{C L}+\frac{T_{a L}}{i_{G B}} \\
v_{M}: \text { motor speed } \\
t_{a}: \text { acceleration time } \\
\eta_{G}: \text { guide efficiency }
\end{array}
$$

During the generation procedure prohibited parameter areas (taboo zones) were calculated to decrease the number of the possible solutions. To set up these zones compatibility functions were applied. The compatibility functions are listed in Table 2

The object of this project was to find the optimal parameters of the linear drive components (servo motor, coupling, gearbox, guide). The study runs on three different robot axes with different boundary conditions (e.g. Brake is required on the selected axis?). The variant parameters at the servo motor are evaluated by using the following fuzzy membership functions (see Figs. 13, 14, 15, 16.

Fig. 11. The arrangement of a robot axi

\section{A MOTOR + COUPLING \\ $2: 5+$ GEARBOX}

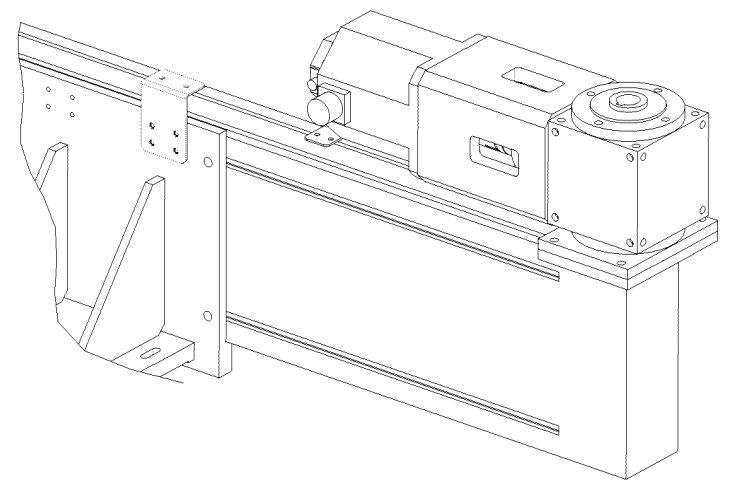

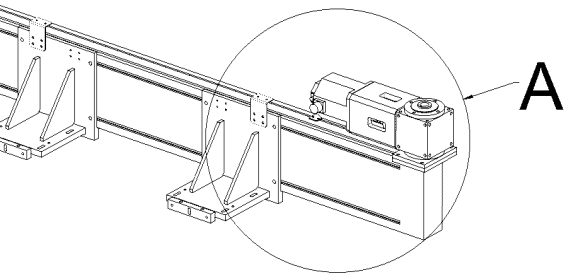

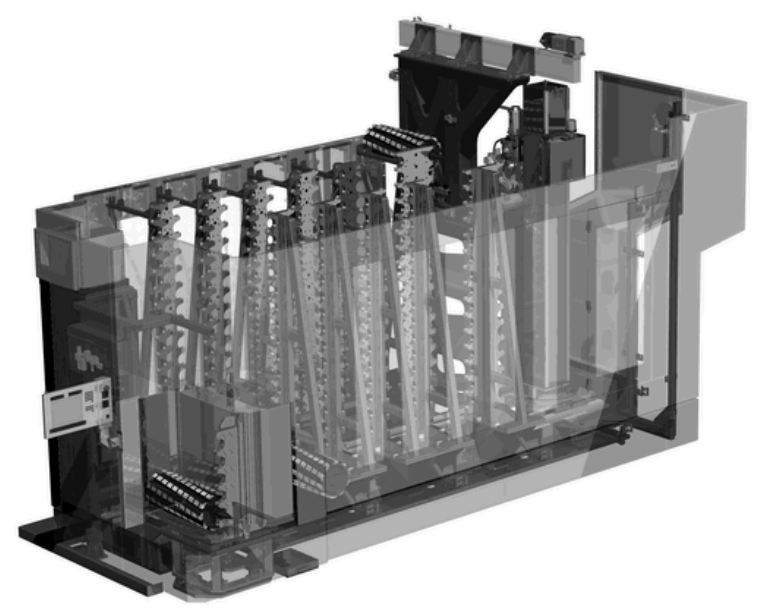

Fig. 12. Tool magazine

The parameter variant fuzzy values are only calculated outside the prohibited parameter areas. The results are organized into a 6dimensional matrix. By fixing 3 coordinates there is a good opportunity to visualize a 3dimensional subset from this matrix. Figs. 17 and 18 show the selected portion of the matrix with the different fuzzy values and the taboo zones (in colour light) in cases of $\mathrm{Y}$ and $\mathrm{Y}$ axes of robot.

The current method (S2) described in this paper is the evolution of a previous study (S1). The original study uses the same fuzzy evaluation method to automatically select existing components for these linear drive systems. The current method uses generalized models instead of existing data. The following two tables (Table 3 and Table 4) show the parameters of the best solutions. 
Tab. 2. The compatibility functions of this project

\begin{tabular}{ll}
\hline ID & Compatibility test between these values: \\
\hline $\mathrm{CP}_{1}\left(\mathrm{a}_{1}, \mathrm{~b}_{1}\right)$ & Motor/Coupling shaft diameter $\left(\mathrm{a}_{1}=\mathrm{d}_{M}, \mathrm{~b}_{1}=\mathrm{d}_{C}\right)$ \\
$\mathrm{CP}_{2}\left(\mathrm{a}_{2}, \mathrm{~b}_{2}\right)$ & $\begin{array}{l}\text { Motor/Coupling torque in case of acceleration } \\
\left(\mathrm{a}_{2}=\mathrm{T}_{M}, \mathrm{~b}_{2}=\mathrm{T}_{C}\right)\end{array}$ \\
$\mathrm{CP}_{3}\left(\mathrm{a}_{3}, \mathrm{~b}_{3}\right)$ & Motor/Gearbox torque value $\left(\mathrm{a}_{3}=\mathrm{T}_{M}, \mathrm{~b}_{3}=\mathrm{T}_{G}\right)$ \\
$\mathrm{CP}_{4}\left(\mathrm{a}_{4}, \mathrm{~b}_{4}\right)$ & Coupling/Gearbox type $\left(\mathrm{a}_{4}=I \mathrm{I}_{M}, \mathrm{~b}_{4}=I \mathrm{D}_{G}\right)$ \\
$\mathrm{CP}_{5}\left(\mathrm{a}_{5}, \mathrm{~b}_{5}\right)$ & Gearbox/Load torque value $\left(\mathrm{a}_{5}=\mathrm{T}_{G}, \mathrm{~b}_{5}=\mathrm{T}_{L}\right)$ \\
$\mathrm{CP}_{6}\left(\mathrm{a}_{6}, \mathrm{~b}_{6}\right)$ & Guide/Axis type $\left(\mathrm{a}_{6}=I \mathrm{I}_{G}, \mathrm{~b}_{6}=I \mathrm{D}_{A}\right)$ \\
\hline
\end{tabular}

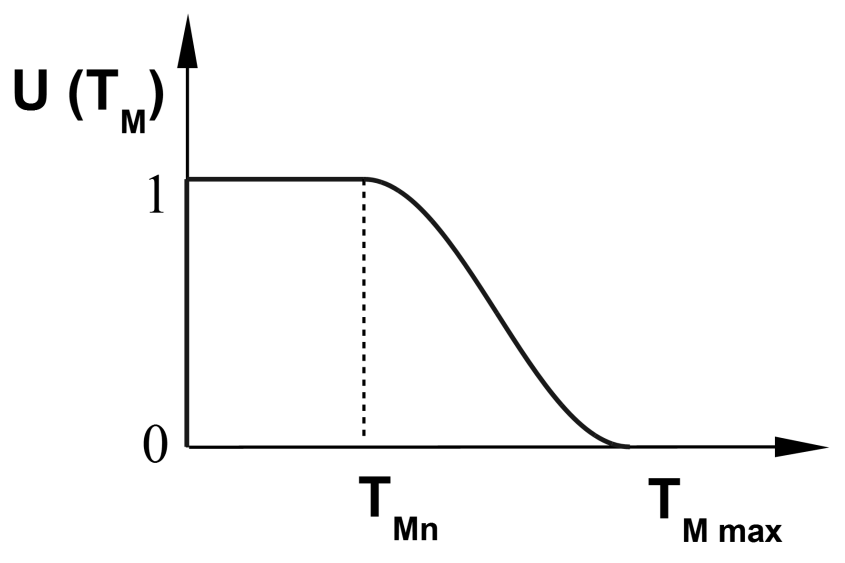

Fig. 13. Fuzzy membership function for maximum/stall torque of the servo motor

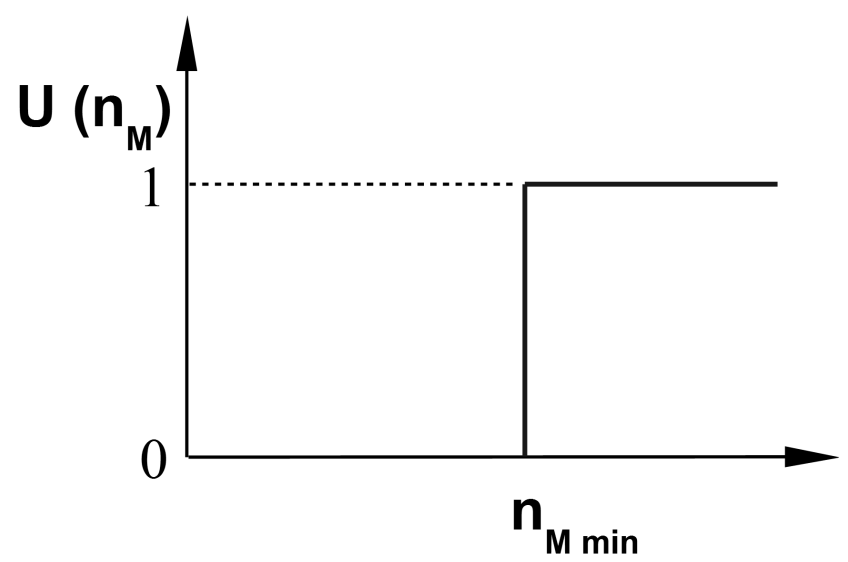

Fig. 14. Fuzzy membership function for the speed of the servo motor

According to study S2, by applying a bigger motor with smaller gearbox ratio both the load speed and the quality of this solution will increase.

According to study S2, by marginally decreasing the load speed and the gearbox ratio a smaller motor is applicable with higher quality of this solution.

\section{Summary}

This paper clearly shows that fuzzy evaluation is definitely an effective method to calculate the optimal parameters of the design. Fuzzy method has the significant advantage of handling

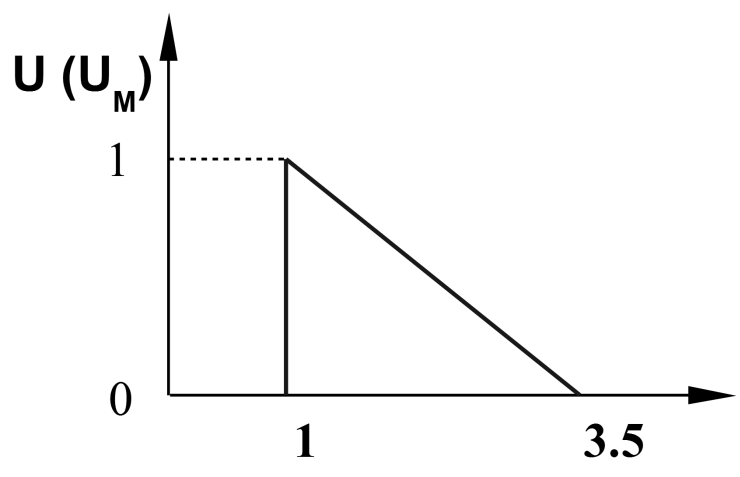

Fig. 15. Fuzzy membership function for utilization ratio of the servo motor

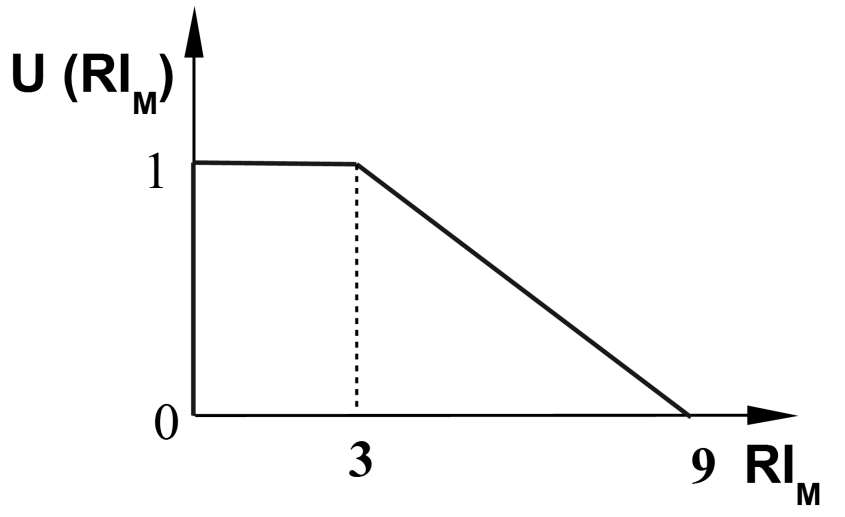

Fig. 16. Fuzzy membership function for inertia ratio of the servo motor

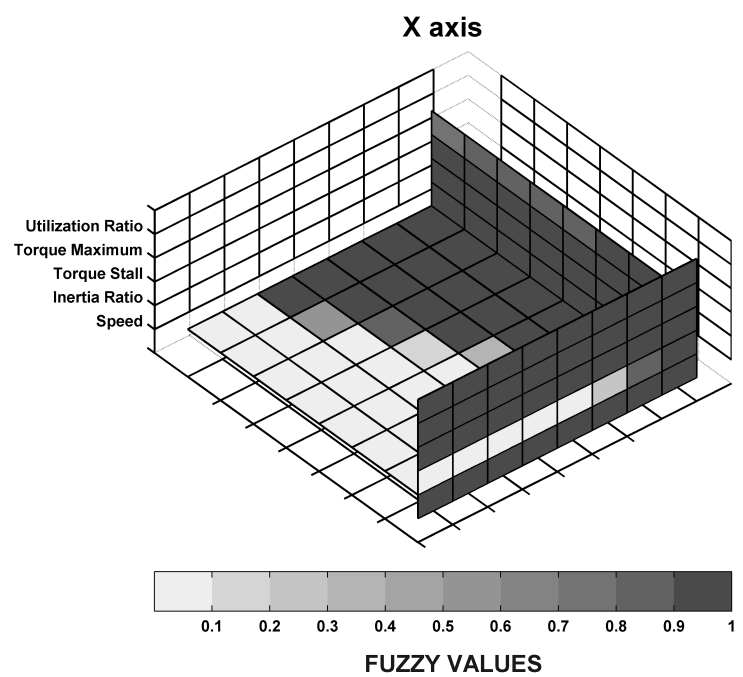

Fig. 17. Fuzzy values in the solution space with light taboo zones at the $\mathrm{X}$ axis of the robot

the inaccurate values both in the values of design parameters and the requirements.

The introduction of the generalized function/component models brings some new opportunities in handling the unknown parameter values. This mathematical method is founded on real data of realized functions. Therefore the calculated intermediate values are realistic. In this way the parameters of the optimized 


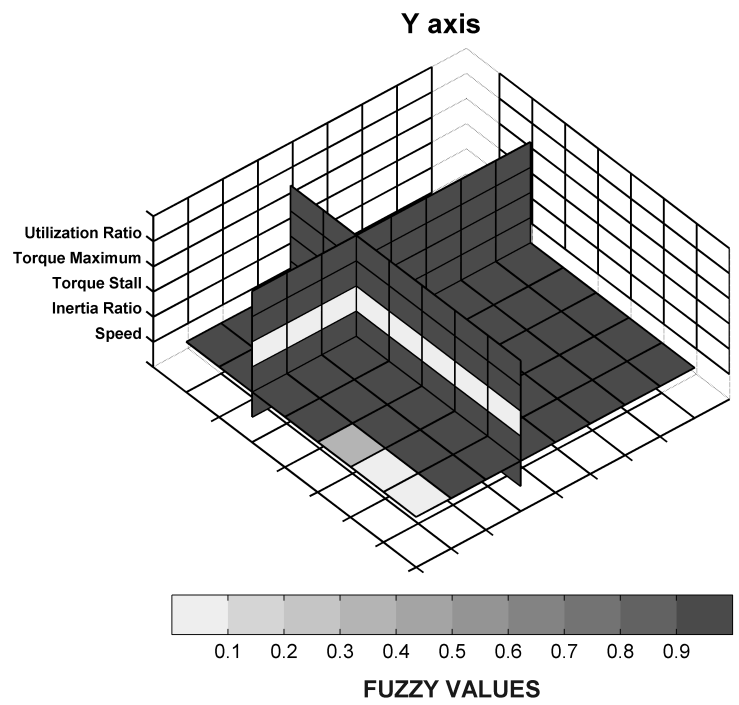

Fig. 18. Fuzzy values in the solution space with light taboo zones at the $\mathrm{Y}$ axis of the robot

Tab. 3. The comparison of the results of S1, S2 studies at the $\mathrm{X}$ axis of the robot

\begin{tabular}{lll}
\hline Result name & S1 & S2 \\
\hline Variant quality - $\mathrm{Q}_{V A R}$ & 0.91263 & 0.93112 \\
Load Speed & 1.6667 & 2.2222 \\
Load Inertia Ratio & 6.8784 & 6.3333 \\
Constant Load Torque at Motor & 0.37462 & 0.39716 \\
Motor Stall Torque & 5.9796 & 7.4724 \\
Acceleration Torque at Motor & 3.2213 & 5.7763 \\
Motor Maximum Torque & 21.1257 & 31.2292 \\
Gearbox ratio & 8 & 6 \\
\hline
\end{tabular}

function are close to the realizable ones.

The method can further be developed into two specific directions. On the one hand, new development trends can be forecast with extrapolation of the generalized models. It requires higher number of initial data and more accurate spline fitting of the functions. On the other hand, the fuzzy evaluation can be supported by other numeric methods like neural networks and genetic algorithms.
Tab. 4. The comparison of the results of S1, S2 studies at the $\mathrm{Y}$ axis of the robot

\begin{tabular}{lll}
\hline Result name & S1 & S2 \\
\hline Variant quality - $Q_{V A R}$ & 0.94817 & 0.96745 \\
Load Speed & 1.25 & 1.1963 \\
Load Inertia Ratio & 1.0937 & 1.25 \\
Constant Load Torque at Motor & 8.0421 & 5.8251 \\
Motor Stall Torque & 8.6043 & 5.8817 \\
Acceleration Torque at Motor & 9.0745 & 6.69 \\
Motor Maximum Torque & 39.3192 & 20.7618 \\
Gearbox ratio & 10 & 8.9167 \\
\hline
\end{tabular}

\section{References}

1 Deciu E R, Ostrosi E, Ferney M, Gheorghe M, Configurable product design using multiple fuzzy models, Journal of Engineering Design 16 (2005), no. 2 .

2 Dombi J, Gera Zs, Rule based fuzzy classification using squashing functions, Journal of Intelligent \& Fuzzy Systems, Applications in Engineering and Technology 19 (2008), no. 1.

3 Vajna S, Kittel K, Bercsey T, Designing the solution space for the autogenetic design theory (ADT), International Design Conference - Design 2010, Dubrovnik.

4 Barajas M, Agard B, A ranking procedure for fuzzy decision-making in product design, International Journal of Production Research, IDMME - Virtual Concept 2008, Beijing. 